\title{
Chemoprevention of premalignant and malignant lesions of oral cavity: Recent trends
}

\author{
Ashish S. Bodhade ${ }^{1}$, Alka M. Dive ${ }^{1}$ \\ 'Department of Oral and Maxillofacial Pathology, \\ VSPM'S Dental College and Research Center, Digdoh \\ Hills, Hingana Road, Nagpur, Maharashtra, India
}

Correspondence: Dr. Ashish S. Bodhade

Email: drbodhade55@rediffmail.com

\begin{abstract}
The word chemoprevention includes prevention of initiation, promotion, and progression of carcinogenesis to cancer. This article is an attempt to review the dietary chemopreventive agents and their mode of action in chemoprevention of oral premalignant lesions and oral cancer using a systematic approach. Selected chemoprevention trials are discussed with a focus on strategies of trial design and clinical outcome. Future in the field of chemoprevention will be more promising than the recently available therapeutic alternatives.
\end{abstract}

Key words: Antioxidants, dietary chemopreventive agents, head and neck malignancy, premalignant lesions

\section{INTRODUCTION}

The word chemoprevention includes prevention of initiation, promotion, and progression of carcinogenesis to cancer. Michael B. Sporn coined the term chemoprevention in a 1976 article in Federation Proceedings, when writing about the work his group was doing with vitamin A analogs. ${ }^{[1]}$ This definition was in contrast with the use of the term "chemotherapy" by Professor Paul Ehrlich in the early $20^{\text {th }}$ century to describe a synthetic agent employed to treat disease. ${ }^{[2]}$

Cancers of the oral cavity represent the sixth most prevalent cancer worldwide. ${ }^{[3]}$ Oral cancer is a major health problem in some parts of the world, especially in developing countries. The age-adjusted incidence rates of oral cancer vary from over 20 per 100,000 populations in India to 10 per 100,000 in the US, and less than 2 per 100,000 in the Middle East. ${ }^{[3]}$ The overall prognosis for these patients is poor with a 5 -year survival rate of $\sim 50 \%$ that has not changed over the last three decades. ${ }^{[3]}$ Attention has focused on understanding the molecular mechanisms of human oral cancer development. Chemoprevention is a promising treatment strategy. Topical and systemic use of chemo preventive agents is an attractive alternative that reduces toxic effects. Over a few decades, many alternative chemopreventive agents were studied for breast cancer and other organs, but very few studies have been carried out on oral premalignant lesions and oral cancer. This article is an attempt to review some chemotherapeutic agents and their mode of action in chemoprevention of oral premalignant lesions and oral cancer.

\section{MOLECULAR ASPECT OF VARIOUS CHEMOPREVENTIVE AGENTS}

Cancer stem cells give rise to the tumor bulk through continuous self-renewal and differentiation. Understanding the mechanisms that regulate self-renewal is of greatest importance for discovery of anticancer drugs targeting cancer stem cells. Foods of plant origin frequently contain several minor dietary nonnutrient compounds. The nonnutrient inhibitors of carcinogenesis have several different mechanisms of action. Some are blocking agents, i.e., they prevent carcinogens from reaching or reacting with critical target sites. Others are suppressing agents, i.e., they

How to cite this article: Bodhade AS, Dive AM. Chemoprevention of premalignant and malignant lesions of oral cavity: Recent trends.

Eur J Dent 2013;7:246-50. 
prevent evolution of the neoplastic process in cells that otherwise would become malignant. Occasionally, one compound will show both mechanisms. Thus, it may be possible to evaluate their impact on cancer risk. ${ }^{[4]}$

The dietary compounds including curcumin, sulforaphane, soy isoflavone, epigallocatechin-3gallate, resveratrol, lycopene, piperine, and vitamin D (3) are suggested for their direct or indirect effect on these self-renewal pathways. Curcumin and piperine have been demonstrated to target breast cancer stem cells. Sulforaphane has been reported to inhibit pancreatic tumor-initiating cells and breast cancer stem cells. These studies provide a basis for preclinical and clinical evaluation of dietary compounds for chemoprevention of cancer stem cells. This may enable us to discover more preventive strategies for cancer management by reducing cancer resistance and recurrence and improving patient survival. ${ }^{[5]}$

Some of the known agents are black raspberries, ${ }^{[6]}$ tretinoin "biofilm," ${ }^{[7]}$ vitamins $\mathrm{A}, \mathrm{E}$, and $\mathrm{C},{ }^{[8]}$ genistein, ${ }^{[9]}$ Spirulina extract, ${ }^{[10]}$ protocatechuic acid and costunolid, ${ }^{[11]}$ black tea polyphenols, ${ }^{[12]}$ and curcumin. ${ }^{[13]}$

\section{Black raspberries}

Lyophilized black raspberries, given in the diet, were found to inhibit esophageal and colon cancer. Casto, et al. (2002) studied the components of lyophilized black raspberries (ellagic and ferulic acids, $\beta$-sitosterol) and berry extract for their ability to selectively inhibit replication of cultured human tumor cells and for their effect on cell cycle progression. ${ }^{[6]}$ Berry extract, ferulic acid, and $\beta$-sitosterol inhibited growth of premalignant and malignant cells, but had no effect on normal oral cells, whereas ellagic acid equally inhibited all cell types. Ferulic acid and $\beta$-sitosterol inhibited progression of the cell cycle at the M/G1 interface, but berry extract appeared not to have any specific effect on stages of the cell cycle. They proposed clinical trials in human oral cancer, including a pre-surgical model to examine the effect of berries on gene expression in oral tumors and a post-surgical model to prevent oral tumor recurrence and for inhibition or modulation of progression of premalignant lesions. ${ }^{[6]}$

\section{Tretinoin biofilm}

Wang, et al. first studied the use of mucosal adhesive film (MAF)/tretinoin biofilm. Their study is based on the hypothesis that topical application of MAF, as a means to deliver tretinoin, is effective and safe for oral cancer chemoprevention in the hamster model. ${ }^{[7]}$
Supplemental and dietary vitamin $\mathrm{E}, \boldsymbol{\beta}$-carotene, and vitamin $C$ intake

Vitamin $\mathrm{E}, \beta$-carotene, and vitamin $\mathrm{C}$ are micronutrient antioxidants that protect cells from oxidative damage involved in prostate carcinogenesis. In separate trials, supplemental vitamin E was associated with a decreased risk of prostate cancer among smokers and supplemental $\beta$-carotene was associated with a decreased risk of prostate cancer among men with low baseline plasma $\beta$-carotene levels. ${ }^{[8]}$ Kaugars $^{[14]}$ and Kirsh, et al..$^{[15]}$ found that their results do not provide strong support for population-wide implementation of high-dose antioxidant supplementation for the prevention of prostate cancer. However, vitamin E supplementation in male smokers and $\beta$-carotene supplementation in men with low dietary $\beta$-carotene intake were associated with reduced risk of this disease. Neuhouser, et al. ${ }^{[16]}$ concluded in their study that plant foods have an important preventive influence in a population at high risk for lung cancer. However, persons who use $\beta$-carotene supplements do not benefit from the protective compounds in plant foods. Heinonen, et al. conducted a study on participants receiving vitamin $A / \beta$-carotene and found protective effect before initiation and accelerating for lung cancer who already developed. ${ }^{[17]}$

\section{Spirulina platensis and fusiformis}

The blue-green microalgae Spirulina, used in daily diets by natives of Africa and America, have been found to be a rich natural source of proteins, carotenoids, and other micronutrients. Experimental studies in animal models have demonstrated an inhibitory effect of Spirulina algae on oral carcinogenesis. Grawish found $S$. platensis is an adjunctive means to inhibit the dysplastic changes occurring in the hamster cheek pouch mucosa. ${ }^{[10]}$

Mathew, et al. (1995) evaluated the chemopreventive activity of Spirulina fusiformis ( $1 \mathrm{~g} /$ day for 12 months) in reversing oral leukoplakia in pan tobacco chewers in Kerala, India. They observed complete regression of lesions in 20 of $44(45 \%)$ subjects supplemented with S. fusiformis, as opposed to 3 of $43(7 \%)$ in the subjects on placebo $(P<0.0001)$. When stratified by the type of leukoplakia, the response was more pronounced in homogeneous lesions: Complete regression was seen in 16 of $28(57 \%)$ subjects with homogeneous leukoplakia, 2 of 8 with erythroplakia, 2 of 4 with verrucous leukoplakia, and 0 of 4 with ulcerated and nodular lesions. ${ }^{[17]}$ Within 1 year of discontinuing supplements, 9 of 20 (45\%) complete responders with $S$. fusiformis developed recurrent 
lesions. Supplementation with S. fusiformis neither resulted in increased serum concentration of retinol or $\beta$-carotene nor was associated with toxicity. ${ }^{[18]}$

\section{Green tea and black tea}

Tea made from young leaves and leaf buds of the tea plant (Camellia sinensis) is the world's second most consumed beverage. Tea polyphenols are strong antioxidants and tea preparations have inhibitory activity against tumorigenesis. There are three main types of tea, all coming from the tea plant, viz. black tea (fermented), green tea (unfermented), and oolong tea (semi-fermented), classified based on the methods of brewing and processing. Inhibition of tumorigenesis by green or black tea preparations has been demonstrated in various animal models in different organs and various epidemiological studies. Still there are no clear conclusions pertaining to the protective effects of tea consumption against cancer development in humans. Many mechanisms have been proposed for the inhibition of carcinogenesis by tea, including the modulation of signal transduction pathways [including growth factor-mediated, mitogen-activated protein kinase (MAPK)-dependent, and ubiquitin/proteasome degradation pathways] that lead to the inhibition of cell proliferation and transformation, induction of apoptosis of preneoplastic and neoplastic cells, and inhibition of tumor invasion as well as angiogenesis. ${ }^{[12,19,20]} \mathrm{Hsu}$, et al. (2002) studied the effects of green tea extract, green tea polyphenols, and the most potent green tea polyphenol, epigallocatechin-3-gallate, on normal human keratinocytes and oral carcinoma cells. ${ }^{[21]}$ The results showed that green tea and its constituents selectively induce apoptosis only in oral carcinoma cells, while epigallocatechin-3-gallate was able to inhibit the growth and invasion of oral carcinoma cells. These differential responses to green tea and its constituents between normal and malignant cells were correlated with the induction of p57, a cell cycle regulator. These data suggest that the chemopreventive effects of green tea polyphenols may involve a p57-mediated survival pathway in normal epithelial cells, while oral carcinoma cells undergo an apoptotic pathway. ${ }^{[21]}$

\section{Genistein}

Genistein is an isoflavone present in soy at high concentrations. It is known to be a natural agent used for prophylaxis and treatment of cancer. ${ }^{[6]}$ Genistein act as an anti-apoptotic agent, causes tumor cell differentiation, inhibits cell proliferation and angiogenesis, modulates cell cycle, exerts antioxidant effect, and acts as an immunosuppressant. It is also used in osteoporosis, cardiovascular disorders, and menopause. All these effects occur by varied concentrations of genistein in diet or by supplementation. ${ }^{[22,23]}$

\section{Curcumin}

Curcumin is the principal curcuminoid of the popular Indian spice, turmeric, which is a member of the ginger family (Zingiberaceae). Turmeric's other two curcuminoids are desmethoxycurcumin and bis-desmethoxycurcumin. The curcuminoids are natural phenols that are responsible for the yellow color of turmeric. It suggests that curcumin has the potential as the leading compound for anti-cancer proliferation and invasion in oral cancer treatment. cdc27, epidermal growth factor receptor (EGFR) substrate 15, peroxisome proliferator-activated receptors-alpha (PPAR-alpha) and H2A histone may also play an important role as anticancer agents. ${ }^{[23,24]}$ Sharma, et al. (2006) demonstrated for the first time that curcumin downregulates smokeless tobacco extract or nicotine-derived nitrosamine ketone (NNK) induced Nuclear Factor-KappaB (NF-kB) and cyclooxygenase-2 in oral premalignant and cancer cells in vitro. ${ }^{[25]}$

\section{Piperine and resveratrol}

Piperine has found to inhibit human CYP3A4 and P-glycoprotein, enzymes important for the metabolism and transport of xenobiotics and metabolites. By inhibiting drug metabolism, piperine may increase the bioavailability of various compounds and alter the effectiveness of some medications. Notably, piperine may enhance bioavailability of curcumin by $2000 \%$ in humans. The exact mechanism of piperine bioavailability enhancing abilities is unknown. Piperine can enhance the pharmacokinetic parameters of resveratrol via inhibiting its glucuronidation, thereby slowing its elimination. Animal studies showed that piperine may extend its chemopreventive effect by modulating lipid peroxidation and augmenting antioxidant defense system. ${ }^{[26]}$

Resveratrol and quercetin are polyphenols which have been detected in significant amounts in green vegetables, citrus fruits, and red grape wines. Beneficial effects attributed to these compounds include anti-inflammatory, antiviral, and antitumor properties. Resveratrol and a combination of resveratrol and quercetin, in concentrations equivalent to that present in red wines, are effective inhibitors of oral squamous carcinoma cell growth and proliferation. ${ }^{[17,27,28]}$ 


\section{Protocatechuic acid and costunolid}

Protocatechuic acid (3,4-dihydroxybenzoic acid) is a simple phenolic compound widely distributed in nature. Like many other simple phenolic acids, protocatechuic acid is detected in almost all plants and is, therefore, a very common component of human diet, such as the bran and grain brown rice (Oryza sativa L.) and onion (Allium cepa L.), especially in the scales. ${ }^{[11]}$ The mechanism of the preventive action of protocatechuic acid is based on its antioxidant property, i.e., inhibition of the generation of free radicals, and ability to scavenge the free radicals and increase the catalytic activity of endogenous enzymes involved in the neutralization of free radicals. It is important that the impact of protocatechuic acid on the activity of enzymes involved in Phase I and II biotransformation of carcinogens and, possibly, direct blocking of specific binding sites of carcinogens with DNA molecule. Other aspects regarding the impact on the activity of cyclooxygenase-2, inducible nitric oxide synthase (iNOS), inflammatory cytokines, and the proteins regulating cell cycle process are poorly understood. ${ }^{[29]}$ Suzuki, et al. (2003) suggested that dietary protocatechuic acid inhibits progression of 4-nitroquinoline 1-oxide induced oral carcinogenesis, and such inhibition might be related to suppression of cell proliferation by protocatechuic acid..$^{[30]}$

\section{Lycopene}

Lycopene (from the New Latin word Lycopersicum for the name of tomato species) is a bright red carotene and carotenoid pigment. It is a phytochemical found in tomatoes and other red fruits and vegetables, such as red carrots, red bell peppers, watermelons, and papayas (but not strawberries or cherries). Although lycopene is chemically a carotene, it has no vitamin A activity. Orly Livny, et al. studied the role of lycopene and $\beta$-carotene and found that lycopene strongly and dose dependently inhibited proliferation of KB-1 human oral tumor cells. $\beta$-Carotene was a far less-effective growth inhibitor. ${ }^{[31]}$ The results of their study further supported the hypothesis that carotenoids, in general, and lycopene, in particular, may be effective anticarcinogenic agents in oral carcinogenesis. Edward Giovannucci, et al. (1999) in their review suggested that the evidence for their benefit was the strongest for cancers of the prostate, lung, and stomach. Data were also suggestive of their benefit for cancers of the pancreas, colon, and rectum, esophagus, oral cavity, breast, and cervix. Because the data are from observational studies, a cause-effect relationship cannot be established definitively. Further, numerous other potentially beneficial compounds are present in tomatoes and their complex interactions among multiple components may contribute to the anticancer properties of tomatoes. ${ }^{[32,33]}$

Tsao, et al. (2004) summarized that patients with head and neck premalignant changes consist of a diverse population and should be treated differently depending on their molecular genotype. Patients with minimal genetic changes may be treated with single-agent retinoids or other agents. Those with more accumulated genetic changes will require combination chemoprevention therapies. Lesions that have advanced genetic changes with mutant p53 may benefit from targeted p53 therapy, and those lesions that express EGFR and COX-2 may require inhibitors of EFGR and COX-2. Other strategies include the oncolytic adenovirus d11520 (ONYX-015), which selectively targets p53-deficient cells. Ongoing trials and future strategies include studying EGFR inhibitors, vascular endothelial growth factor receptor (VEGF-R) inhibitors, demethylating agents, farnesyltransferase inhibitors, celecoxib, vitamin E, and Bowman-Birk inhibitors. ${ }^{[34]}$

\section{CONCLUSION}

There are numerous chemopreventive agents present in our daily diet which exert their antimutagenic action to routinely exposed mutagens. But in patients with premalignant lesions and cancer, these agents might remain short in their action. These agents might offer cure for malignancy and reversal of premalignancy in a specific concentration. Controlled clinical animal and human trials should be conducted in future to study the specific action of these chemopreventive agents.

\section{REFERENCES}

1. Theisen C. Chemoprevention: What's in a Name? J Natl Cancer Inst 2001;93:743.

2. Jordan VC: Estrogen, selective estrogen receptor modulation, and coronary heart disease: Something or nothing. J Natl Cancer Inst 2001;93:2-4.

3. Mehrotra R, Pandya S, Chaudhary AK, Kumar M, Singh M. Prevalence of oral pre-malignant and malignant lesions at a Tertiary Level Hospital in Allahabad, India. Asian Pac J Cancer Prev 2008;9:263-5.

4. Wattenberg LW. Inhibition of Carcinogenesis by Minor Dietary Constituents. Cancer Res 1992;52:2085s-91.

5. Li Y, Wicha MS, Schwartz SJ, Sun D. Implications of cancer stem cell theory for cancer chemoprevention by natural dietary compounds. J Nutr Biochem 2011;22:799-806.

6. Casto BC, Kresty LA, Kraly CL, Pearl DK, Knobloch TJ, Schut HA, et al. Chemoprevention of oral cancer by black raspberries. Anticancer Res 2002;22:4005-15.

7. Wang Z, Polavaram R, Fuentes CF, Shapshay SM. Topical Chemoprevention of Oral Cancer with Tretinoin "Biofilm". Arch Otolaryngol Head Neck Surg 2003;129:869-73. 
8. Mamede AC, Tavares SD, Abrantes AM, Trindade J, Maia JM, Botelho MF. The role of vitamins in cancer: A review. Nutr Cancer 2011;63:479-94.

9. Yang Y, Zhou ZT, Ge JP. Effect of genistein on DMBA-induced oral carcinogenesis in hamster. Carcinogenesis 2006;27:578-83.

10. Grawish ME. Effects of Spirulina platensis extract on Syrian hamster cheek pouch mucosa painted with 7,12-dimethylbenz[a]anthracene. Oral Oncol 2008;44:956-62.

11. Ohnishi M, Yoshimi N, Kawamori T, Ino N, Hirose Y, Tanaka T, et al. Inhibitory effects of dietary protocatechuic acid and costunolide on 7,12-dimethylbenz[a]anthracene-induced hamster cheek pouch carcinogenesis. Jpn J Cancer Res 1997;88:111-9.

12. Mujtaba T, Dou QP. Black tea polyphenols inhibit tumor proteasome activity. In Vivo 2012;26:197-202.

13. Chen JW. Anti-proliferative and anti-metastatic effects of curcumin on oral cancer cells. Hua Xi Kou Qiang Yi Xue Za Zhi 2011;29:83-6.

14. Kaugars GE, Silverman S Jr, Lovas JG, Brandt RB, Riley WT, Dao Q, et al. A clinical trial of antioxidant supplements in the treatment of oral leukoplakia. Oral Surg Oral Med Oral Pathol 1994;78:462-8.

15. Kirsh VA, Hayes RB, Mayne ST, Chatterjee N, Subar AF, Dixon LB, et al. Supplemental and dietary vitamin E, beta-carotene, and vitamin C intakes and prostate cancer risk. J Natl Cancer Inst 2006;98:245-54.

16. Neuhouser ML, Patterson RE, Thornquist MD, Omenn GS, King IB, Goodman GE. Fruits and vegetables are associated with lower lung cancer risk only in the placebo arm of the beta-carotene and retinol efficacy trial (CARET). Cancer Epidemiol Biomarkers Prev 2003;12:350-8.

17. The effect of vitamin $\mathrm{E}$ and beta carotene on the incidence of lung cancer and other cancers in male smokers. The Alpha-Tocopherol Beta Carotene Cancer Prevention Study Group. N Engl J Med 1994; 330:1029-35.

18. Mathew B, Sankaranarayanan R, Nair PP, Varghese C, Somanathan T, Amma BP, et al. Evaluation of chemoprevention of oral cancer with Spirulina fusiformis. Nutr Cancer 1995;24:197-202.

19. Shukla Y. Tea and cancer chemoprevention: A comprehensive review. Asian Pac J Cancer Prev 2007;8:155-66.

20. Lee UL, Choi SW. The chemopreventive properties and therapeutic modulation of green tea polyphenols in oralsquamous cell carcinoma. ISRN Oncol 2011;2011:403707.

21. Hsu SD, Singh BB, Lewis JB, Borke JL, Dickinson DP, Drake L, et al. Chemoprevention of oral cancer by green tea. Gen Dent 2002; 50:140-6.

22. Ho YC, Yang SF, Peng CY, Chou MY, Chang YC. Epigallocatechin -3-gallate inhibits the invasion of human oral cancer cells and decreases the productions of matrix metalloproteinases and urokinase-plasminogen activator. J Oral Pathol Med 2007;36:588-93.
23. Polkowski K, Mazurek AP. Biological properties of genistein a review of in vitro and in vivo data. Acta Pol Pharm 2000;57:135-55.

24. Elattar TM, Virji AS. The inhibitory effect of curcumin, genistein, quercetin and cisplatin on the growth of oral cancer cells in vitro. Anticancer Res 2000;20:1733-8.

25. Sharma C, Kaur J, Shishodia S, Aggarwal BB, Ralhan R. Curcumin down regulates smokeless tobacco-induced NF- $\mathrm{kB}$ activation and COX-2 expression in human oral premalignant and cancer cells. Toxicology 2006;228:1-15.

26. Selvendiran K, Singh JP, Krishnan KB, Sakthisekaran D. Cytoprotective effect of piperine against benzo[a]pyrene induced lung cancer with reference to lipid peroxidation and antioxidant system in Swiss albino mice. Fitoterapia 2003;74:109-15.

27. ElAttarTM, Virji AS. Modulating effect of resveratrol and quercetin on oral cancer cell growth and proliferation. Anticancer Drugs 1999;10:187-93.

28. Elattar TM, Virji AS. The effect of red wine and its components on growth and proliferation of human oral squamous carcinoma cells. Anticancer Res 1999;19:5407-14.

29. Tanaka T, Tanaka T, Tanaka M. Potential Cancer Chemopreventive Activity of Protocatechuic Acid. J Exp Clin Med 2011;3:27-33.

30. Suzuki R, Kohno H, Sugie S. Dietary protocatechuic acid during the progression phase exerts chemopreventive effects on chemically induced rat toungue carcinogenesis. Asian Pac J Cancer Prev 2003;4:319-26.

31. Livny O, Kaplan I, Reifen R, Polak-Charcon S, Madar Z, Schwartz B. Lycopene Inhibits Proliferation and Enhances Gap-Junction Communication of KB-1 Human Oral Tumor Cells. J Nutr 2002;132:123754-9.

32. Giovannucci E. Tomatoes, Tomato-Based Products, Lycopene, and Cancer: Review of the Epidemiologic Literature. J Natl Cancer Inst 1999;9:317-31.

33. Giovannucci E. A Review of Epidemiologic Studies of Tomatoes, Lycopene, and Prostate Cancer. Exp Biol Med 2002;227:10852-9.

34. Tsao AS, Kim ES, Hong WK. Chemoprevention of cancer. CA Cancer J Clin 2004;54:150-80.

\begin{tabular}{|l|l|}
\hline \multicolumn{2}{|c|}{ Access this article online } \\
\hline Quick Response Code: & Website: \\
& www.eurjdent.com \\
\cline { 3 - 4 } & Source of Support: Nil. \\
&
\end{tabular}

\section{“QUICK RESPONSE CODE” LINK FOR FULL TEXT ARTICLES}

The journal issue has a unique new feature for reaching to the journal's website without typing a single letter. Each article on its first page has a "Quick Response Code". Using any mobile or other hand-held device with camera and GPRS/other internet source, one can reach to the full text of that particular article on the journal's website. Start a QR-code reading software (see list of free applications from http://tinyurl.com/yzlh2tc) and point the camera to the QR-code printed in the journal. It will automatically take you to the HTML full text of that article. One can also use a desktop or laptop with web camera for similar functionality. See http://tinyurl.com/2bw7fn3 or http://tinyurl.com/3ysr3me for the free applications. 